\title{
An Integral Analogue of Taylor's Series and Its Use in Computing Fourier Transforms
}

\author{
By Thomas J. Osler
}

Abstract. In this paper, an integral analogue of Taylor's series

$$
f(z)=\int_{-\infty}^{\infty} D^{\omega} f\left(z_{0}\right)\left(z-z_{0}\right) \omega / \Gamma(\omega+1) d \omega
$$

is discussed. $D^{\omega} f(z)$ is a fractional derivative of order $\omega$. Extensions of this integral are also given, one of which is an integral analogue of Lagrange's expansion. These integrals are shown to be generalizations of the Fourier integral theorem. Several special cases of these integrals are computed, and a table of Fourier transforms emerges.

1. Introduction. The fractional derivative of order $\alpha$ of the function $f(z)$ with respect to $g(z), D_{o(z)}^{\alpha} f(z)$, is a generalization of the familiar derivative $d^{\alpha} f(z) /(d g(z))^{\alpha}$ to nonintegral values of $\alpha$. In the author's previous papers on the fractional calculus, three distinct features evolved:

(1) Certain formulas familiar from the elementary calculus were shown to be special cases of more general expressions involving fractional derivatives. These included Taylor's series [5], Leibniz rule [3], [6], [7], the chain rule [4], and Lagrange's expansion [5].

(2) Through the fractional calculus, we were able to relate formulas familiar from the study of Fourier analysis to the above-mentioned calculus relations. Thus, it was shown that the generalized Taylor's series could be viewed as an extension of the Fourier series [5], and that the generalized Leibniz rule was an extension of Parseval's relation [7].

(3) Most of the important special functions can be represented by fractional derivatives of elementary functions, such as

$$
J_{\nu}(z)=\pi^{-1 / 2}(2 z)^{-\nu} D_{z^{2}}^{-\nu-1 / 2}(\cos z) / z .
$$

We found that our extensions of calculus formulas, when combined with fractional derivative representations for the higher functions, produced interesting series relations involving the special functions.

This paper continues our study of the fractional calculus by exposing the three features outlined above for certain integrals which are related to Taylor's series and Lagrange's expansion. We find it useful to distinguish three special cases:

Case 1. The expression

$$
f(z)=\int_{-\infty}^{\infty} \frac{\left.D_{z-b}^{\omega+\gamma} f(z)\right|_{z=z_{0}}}{\Gamma(\omega+\gamma+1)}\left(z-z_{0}\right)^{\omega+\gamma} d \omega
$$

Received May 16, 1971.

AMS 1970 subject classifications. Primary 26A33, 42A68; Secondary 44A45, 33A30, 30A10.

Key words and phrases. Fractional derivative, Fourier integral theorem, Fourier transforms, Taylor series, Lagrange expansion, special functions.

Copyright @ 1972, American Mathematical Society 
is an integral analogue of Taylor's series. Here, $\gamma$ is an arbitrary complex number, and $z$ is restricted to the circle $\left|z-z_{0}\right|=\left|z_{0}-b\right|$. (1.1) is easily suggested by the generalized Taylor's series

$$
f(z)=\sum_{n=-\infty}^{\infty} \frac{\left.a D_{z-b}^{a n+\gamma} f(z)\right|_{z-z_{0}}}{\Gamma(a n+\gamma+1)}\left(z-z_{0}\right)^{a n+\gamma}
$$

by setting $a n=\omega_{n}$ and $\Delta \omega=a$. As $a \rightarrow 0^{+}$, we see at once that this series is the approximating sum in the definition of the Riemann integral for (1.1). The integral (1.1) was mentioned in passing by G. H. Hardy [2, p. 56], but no rigorous derivation appears in the literature, to the best of the author's knowledge.

Case 2. Since the natural relation $D^{a} D^{b} f(z)=D^{a+b} f(z)$ is valid (with certain restrictions on $a, b$, and $f(z)$ ), a simple generalization of (1.1) becomes

$$
D_{z-b}^{\alpha} f(z)=\int_{-\infty}^{\infty} \frac{\left.D_{z-b}^{\alpha+\omega+\gamma} f(z)\right|_{z-z_{0}}}{\Gamma(\omega+\gamma+1)}\left(z-z_{0}\right)^{\omega+\gamma} \underline{d} \omega .
$$

Case 3. Our final relation

$$
f(z)=\int_{-\infty}^{\infty} \frac{\left.D_{z-b}^{\omega+\gamma}\left[f(z) \theta^{\prime}(z) q(z)^{-\omega-\gamma-1}\right]\right|_{z-z_{0}}}{\Gamma(\omega+\gamma+1)} \theta(z)^{\omega+\gamma} d \omega
$$

is an integral analogue of Lagrange's expansion. Here, $\theta(z)=\left(z-z_{0}\right) q(z)$ and the integral is valid for $z$ on the closed curve $|\theta(z)|=|\theta(b)|$. (1.1) is the special case of (1.3) in which $q(z) \equiv 1$ (and thus $\left.\theta(z) \equiv z-z_{0}\right)$. The truth of (1.3) is suggested at once by letting $a \rightarrow 0^{+}$in the generalized Lagrange's expansion [5]

$$
f(z)=\sum_{n=-\infty}^{\infty} \frac{\left.a D_{z-b}^{a n+\gamma}\left[f(z) \theta^{\prime}(z) q(z)^{-a n-\gamma-1}\right]\right|_{z-z_{0}}}{\Gamma(a n+\gamma+1)} \theta(z)^{a n+\gamma} .
$$

(1.2) and (1.3) have not, to the best of the author's knowledge, appeared before in the literature.

We demonstrate in Sections 3 and 4 that (1.1) and (1.3) are generalizations of the familiar Fourier integral theorem.

The paper concludes by examining special cases of (1.1), (1.2), and (1.3) in which specific functions are chosen for $f(z)$ and $\theta(z)$. We find that Fourier transforms of the special functions emerge. These are listed in Table 5.1. Most of the entries in this table appear to be new.

2. Fractional Differentiation. In this section, we briefly state our definition of fractional differentiation so as to make the paper self-contained. The reader unfamiliar with this subject can refer to [3] for a discussion and motivation of the following definition.

Definition 2.1. Let $f(z)$ be analytic in the simply connected region $R$. Let $z=b$ be an interior or boundary point of $R$. Assume that $\oint_{c}|f(z)||d z|$ exists for any simple closed contour $C$ in $R \cup\{b\}$ through $b$. Then, if $\alpha$ is not a negative integer and $z$ is in $R$, we define the fractional derivative of order $\alpha$ of $f(z)$ with respect to $z-b$ to be

$$
D_{z-b}^{\alpha} f(z)=\frac{\Gamma(\alpha+1)}{2 \pi i} \int_{b}^{(z+)} \frac{f(t) d t}{(t-z)^{\alpha+1}}
$$


For nonintegral $\alpha$, the integrand has a branch line which begins at $t=z$ and passes through $t=b$. We define $(t-z)^{\alpha+1}$ to be $\exp [(\alpha+1) \ln (t-z)]$, where $\ln (t-z)$ is real when $t-z$ is positive. The notation on this integral implies that the contour of integration starts at $t=b$, encloses $t=z$ once in the positive sense, and returns to $t=b$ without crossing the branch line or leaving $R \cup\{b\}$. When $\operatorname{Re}(\alpha)<0$, we can replace this closed contour by a line from $b$ to $z$, [3],

$$
D_{z-b}^{\alpha} f(z)=\frac{1}{\Gamma(-\alpha)} \int_{b}^{z} \frac{f(t) d t}{(z-t)^{\alpha+1}} .
$$

This integral is called the Riemann-Liouville integral when $b=0$, and permits us to extend the definition of $D^{\alpha}$ to the case where $\alpha$ is a negative integer.

If we wish to determine the fractional derivative of a specific function, it is convenient to refer to Chapter 13 of [1, Vol. 2] which contains a table of "RiemannLiouville fractional integrals". We note that in our notation the Riemann-Liouville fractional integral of order $\alpha$ of the function $f(z)$ is denoted by $D_{z}^{-\alpha} f(z)$.

3. Intuitive Motivation and Relation to Fourier Analysis. In the introduction, we discussed a simple manner in which the integral analogues of Taylor's series and Lagrange's expansion are suggested by the author's generalized Taylor's and Lagrange's series for fractional derivatives by letting the parameter $a \rightarrow 0^{+}$. In this section, we discuss motivation for the integral analogue of Taylor's series (1.1) by showing how it evolves formally from the Fourier integral theorem. Simultaneously, we discover that our integral analogue of Taylor's series is, in a certain sense, an extension of Fourier's integral theorem.

In our definition of fractional differentiation (2.1),

$$
\left.D_{z}^{\omega} f(z)\right|_{z=z_{0}}=\frac{\Gamma(\omega+1)}{2 \pi i} \int_{0}^{\left(z_{0}+\right)} \frac{f(t) d t}{\left(t-z_{0}\right)^{\omega+1}},
$$

choose the contour of integration to be the circle centered at $z_{0}$ passing through the origin. We introduce the parameter $\xi$ on this circle through the relation

$$
t=z_{0}+z_{0} e^{i \xi}, \quad|\xi|<\pi \text {. }
$$

Thus, we have

$$
\frac{\left.z_{0}^{\omega} D_{z}^{\omega} f(z)\right|_{z-z_{0}}}{\Gamma(\omega+1)}=\frac{1}{2 \pi} \int_{-\pi}^{\pi} f\left(z_{0}+z_{0} e^{i \xi}\right) e^{-i \xi \omega} d \xi .
$$

Since the 1.h.s. of (3.1) is the Fourier transform of the function

$$
\begin{aligned}
f^{*}(\zeta) & =f\left(z_{0}+z_{0} e^{i \zeta}\right), & & |\zeta|<\pi, \\
& =0, & & |\zeta|>\pi,
\end{aligned}
$$

we have at once from the Fourier integral theorem

$$
f^{*}(\zeta)=\int_{-\infty}^{\infty} \frac{\left.D_{z}^{\omega} f(z)\right|_{z-z_{0}} z_{0}^{\omega} e^{i \omega \zeta}}{\Gamma(\omega+1)} d \omega .
$$

If we call

$$
z=z_{0}+z_{0} e^{i 5},
$$


this last expression becomes

$$
f(z)=\int_{-\infty}^{\infty} \frac{\left.D_{z}^{\omega} f(z)\right|_{z-z_{0}}}{\Gamma(\omega+1)}\left(z-z_{0}\right)^{\omega} d \omega
$$

the special case of (1.1) in which $\gamma=0$. In the next section, we will derive rigorously our most general integral (1.3) through a procedure similar to that outlined above for the special integral (3.3). We have selected this special case in order to show clearly that our integrals are generalizations of the Fourier integral theorem because of the introduction of the complex parameter " $z_{0}$ ". Note that each value of $z_{0}$ determines a circle (3.2) in the $z$-plane on which our integral (3.3) converges to $f(z)$. Since, in general, we are free to vary $z_{0}$ over some open set in the complex plane, (3.3) can represent $f(z)$ for $z$ on an open set (determined by (3.2)). We say that we have "extended the Fourier integral theorem into the complex plane".

Previously the author demonstrated that the familiar Fourier series

$$
f^{*}(\zeta)=\sum_{n=-\infty}^{\infty} f_{n} e^{i a n \zeta}
$$

is, in a similar sense, a special case of the formula

$$
f(z)=\sum_{n=-\infty}^{\infty} \frac{\left.a D_{z-b}^{a n+\gamma} f(z)\right|_{z-z_{0}}}{\Gamma(a n+\gamma+1)}\left(z-z_{0}\right)^{a n+\gamma}
$$

and the Parseval's relation

$$
\frac{a}{2 \pi} \int_{-\pi / a}^{\pi / a} u^{*}(\zeta) v^{*}(\zeta) d \zeta=\sum_{n=-\infty}^{\infty} \frac{a}{2 \pi} \int_{-\pi / a}^{\pi / a} u^{*}(\zeta) e^{i a n \zeta} d \zeta \cdot \frac{a}{2 \pi} \int_{-\pi / a}^{\pi / a} v^{*}(\zeta) e^{-i a n \zeta} d \zeta
$$

is a special case of the generalized Leibniz rule

$$
D_{z}^{\alpha} u(z) v(z)=\sum_{n=-\infty}^{\infty}\left(\begin{array}{c}
\alpha \\
a n+\gamma
\end{array}\right) D_{z}^{\alpha-a n-\gamma} u(z) D_{z}^{a n+\gamma} v(z)
$$

Both (3.4) and (3.5) are extensions into the complex plane of familiar formulas from Fourier analysis. Thus, we have shown how another formula, the Fourier integral theorem, can be extended into the complex plane.

4. Rigorous Derivations. In this section, we present rigorous derivations of (1.1), (1.2), and (1.3), whose validity has already been suggested. The hypothesis of the following theorem is nearly identical to that of Theorem 4.1 in [5], which discusses the generalized Taylor's series. The proof of this Theorem is nothing more than a careful generalization of the formal discussion of the previous section centered on the Fourier integral theorem.

THEOREM. Let $\theta(z)$ be a given function such that (i) the curves $C(r)=\{z|| \theta(z) \mid=r\}$ are simple and closed for each $r$ such that $0<r \leqq p$, (ii) $\theta(z)$ is analytic inside and on $C(p)$, and (iii) $\theta(z)=\left(z-z_{0}\right) q(z), z_{0}$ is inside $C(p)$, and $q(z)$ has no zeros inside $C(p)$. Let $b \neq z_{0}$ be a fixed point inside $C(p)$. Let $\theta(z)^{c}=\exp (c \ln \theta(z))$ denote that branch of the function which is continuous and single valued on the region inside $C(p)$ cut by the branch line $z=z_{0}+\left(b-z_{0}\right) r, 0 \leqq r$, such that $\ln \theta(z)$ is real where $\theta(z)>0$. Let $f(z)$ satisfy the conditions of Definition 2.1 for the existence of $D_{z-b}^{\alpha} f(z)$ for $\{z \mid z$ inside $C(p)$; but $\left.z \neq b+r \exp \left(i \arg \left(b-z_{0}\right)\right), 0 \leqq r\right\}$. Then, for arbitrary $\gamma$ and $z$ 
on the curve $C(|\theta(b)|)$,

$$
f(z)=\int_{-\infty}^{\infty} \frac{\left.D_{z-b}^{\omega+\gamma}\left[f(z) \theta^{\prime}(z) q(z)^{-\omega-\gamma-1}\right]\right|_{z-z_{0}}}{\Gamma(\omega+\gamma+1)} \theta(z)^{\omega+\gamma} d \omega .
$$

Proof. Since $\theta(z)$ is analytic and univalent inside and on a simply connected region containing the curve $C(|\theta(b)|)$, we can describe $\theta(z)$ on this curve by $\theta(z)=$ $-\theta(b) e^{i \zeta}$, for $-\pi<\zeta<\pi$. Define

$$
\begin{aligned}
f^{*}(\zeta) & =f\left(\theta^{-1}\left(-\theta(b) e^{i \zeta}\right)\right) e^{-i \zeta \gamma}, & & \text { for }-\pi<\zeta<\pi, \\
& =0, & & \text { otherwise. }
\end{aligned}
$$

The Fourier integral theorem is valid for $f(\zeta)$, since

$$
\int_{-\infty}^{\infty}|f(\zeta)| d \zeta=\int_{-\pi}^{\pi}\left|f\left(\theta^{-1}\left(-\theta(b) e^{i \zeta}\right)\right) e^{-i \zeta \gamma}\right| d \zeta .
$$

This integral is finite, since we require the possible singularity of $f(z)$ at $z=b$ to be such that $\Phi|f(z)||d z|$ exists when the contour passes through $z=b$ in the Definition 2.1. Applying the Fourier integral theorem to $f^{*}(\zeta)$, we get

$$
\begin{aligned}
& f^{*}(\zeta)=\frac{1}{2 \pi} \int_{-\infty}^{\infty} e^{i \zeta \omega} \int_{-\infty}^{\infty} f^{*}(\phi) e^{-i \phi \omega} d \phi d \omega, \\
& f(z) e^{-i \gamma \zeta}= \int_{-\infty}^{\infty} \frac{e^{i \zeta \omega}(-\theta(b))^{\omega+\gamma}}{\Gamma(\omega+\gamma+1)} \\
& \cdot\left\{\frac{\Gamma(\omega+\gamma+1)}{2 \pi i} \int_{-\pi}^{\pi} \frac{f\left(\theta^{-1}(-\theta(b)) e^{i \phi}\right) i(-\theta(b)) e^{i \phi} d \phi d \omega}{(-\theta(b))^{\omega+\gamma+1} e^{i \phi(\omega+\gamma+1)}}\right\}, \\
& f(z)=\int_{-\infty}^{\infty} \frac{\theta(z)^{\omega+\gamma}}{\Gamma(\omega+\gamma+1)} \frac{\Gamma(\omega+\gamma+1)}{2 \pi i} \int_{b}^{\left(z_{0}+\right)} \frac{f(t) \theta^{\prime}(t) d t}{\left(t-z_{0}\right)^{\omega+\gamma+1} q(t)^{\omega+\gamma+1}} d \omega .
\end{aligned}
$$

Comparing this last expression with the definition of fractional differentiation (2.1), we have (4.1) at once.

COROLlaRY. Let $f(z)=(z-b)^{p} g(z)$, where $g(z)$ is analytic in the circular region $R=\{z|| z-b \mid<r\}$. Let $z_{0} \in R$ be such that the circle $C$ defined by $C=\left\{z|| z-z_{0} \mid\right.$ $\left.=\left|b-z_{0}\right|\right\}$ is contained in $R$. Let $p>-1$ and $p-\alpha>-1$. Then

$$
D_{z-b}^{\alpha} f(z)=\int_{-\infty}^{\infty} \frac{\left.D_{z-b}^{\alpha+\omega+\gamma} f(z)\right|_{z-z_{0}}\left(z-z_{0}\right)^{\omega+\gamma}}{\Gamma(\omega+\gamma+1)} d \omega
$$

for $z$ on $C$.

Proof. The conclusion follows at once if we set $\theta(z)=z-z_{0}$ in the Theorem (thus obtaining (1.1)), and replace $f(z)$ by $D_{z-b}^{\alpha} f(z)$, provided

$$
D_{z-b}^{\omega+\gamma}\left(D_{z-b}^{\alpha} f(z)\right)=D_{z-b}^{\alpha+\omega+\gamma} f(z) \text {. }
$$

To see that (4.2) is true, we expand $f(z)$ in powers, for $z \in R$,

$$
f(z)=\sum_{n=0}^{\infty} a_{n}(z-b)^{p+n} .
$$

Since $p>-1$, we can compute 


$$
D_{z-b}^{\alpha} f(z)=\sum_{n=0}^{\infty} \frac{\Gamma(p+n+1) a_{n}(z-b)^{p-\alpha+n}}{\Gamma(p-\alpha+n+1)} .
$$

The validity of this termwise differentiation is well known [3]. We note in particular that since $p$ and $\alpha$ are restricted in the hypothesis by $p>-1$, and $p-\alpha>-1$, (i) both gamma functions appearing in the last expression are defined, and (ii) $D_{z \rightarrow b}^{\alpha} f(z)$ can be differentiated fractionally.

$$
\begin{aligned}
D_{z-b}^{\omega+\gamma}\left(D_{z-b}^{\alpha} f(z)\right) & =\sum_{n=0}^{\infty} \frac{\Gamma(p+n+1) a_{n}(z-b)^{p-\alpha-\omega-\gamma+n}}{\Gamma(p-\alpha-\omega-\gamma+n+1)} \\
& =D_{z-b}^{\alpha+\omega+\gamma} f(z) .
\end{aligned}
$$

Thus, (4.2) is valid and the Corollary is proved.

Having derived our general integrals, we proceed next to an examination of several special examples.

5. Computation of Fourier Transforms. We conclude our discussion of the integral analogue of Taylor's series by selecting specific functions for $f(z), q(z)$, $\theta(z)$, and taking $b=0$ in the three expressions (1.1), (1.2), and (1.3). The fractional derivatives encountered are computed by referring to the Table of Fractional Integrals (Riemann-Liouville integrals) in Chapter 13.1 of [1, Vol. 2, pp. 185-200]. As an example, we set $f(z)=z^{p}$ in (1.1) and obtain

$$
z^{p}=\int_{-\infty}^{\infty} \frac{\left.D_{z}^{\omega+\gamma} z^{p}\right|_{z=z_{0}}}{\Gamma(\omega+\gamma+1)}\left(z-z_{0}\right)^{\omega+\gamma} d \omega .
$$

Using formula 7 in the above mentioned table, we see that

$$
\left.D_{z}^{\omega+\gamma} z^{p}\right|_{z=z_{0}}=\frac{\Gamma(p+1) z_{0}^{p-\omega-\gamma}}{\Gamma(p-\omega-\gamma+1)}
$$

for $\operatorname{Re}(p)>-1$. A little simplification reveals that

$$
\left(z / z_{0}\right)^{p}=\int_{-\infty}^{\infty}\left(\begin{array}{c}
p \\
\omega+\gamma
\end{array}\right)\left(\left(z / z_{0}\right)-1\right)^{\omega+\gamma} d \omega,
$$

where

$$
\left(\begin{array}{l}
a \\
b
\end{array}\right)=\frac{\Gamma(a+1)}{\Gamma(a-b+1) \Gamma(b+1)}
$$

Since this integral is valid only for $z$ on the circle $\left|z-z_{0}\right|=\left|z_{0}\right|$, we set $z=z_{0}+z_{0} e^{-i \phi}$, for $|\phi|<\pi$. We then have

$$
\begin{aligned}
\int_{-\infty}^{\infty}\left(\begin{array}{c}
p \\
\omega+\gamma
\end{array}\right) e^{-i \phi(\omega+\gamma)} d \omega & =\left(1+e^{-i \phi}\right)^{p}, & & |\phi|<\pi, \\
& =0, & & |\phi|>\pi .
\end{aligned}
$$

(We recall from the proof of the Theorem that our integral is zero for $|\phi|>\pi$.) Since $\gamma$ is an arbitrary complex number, we can write an equivalent expression for this last integral by setting $\gamma=0$, and replacing the path of integration by a horizontal line through $i \eta$ ( $\eta$ any real number). We obtain 


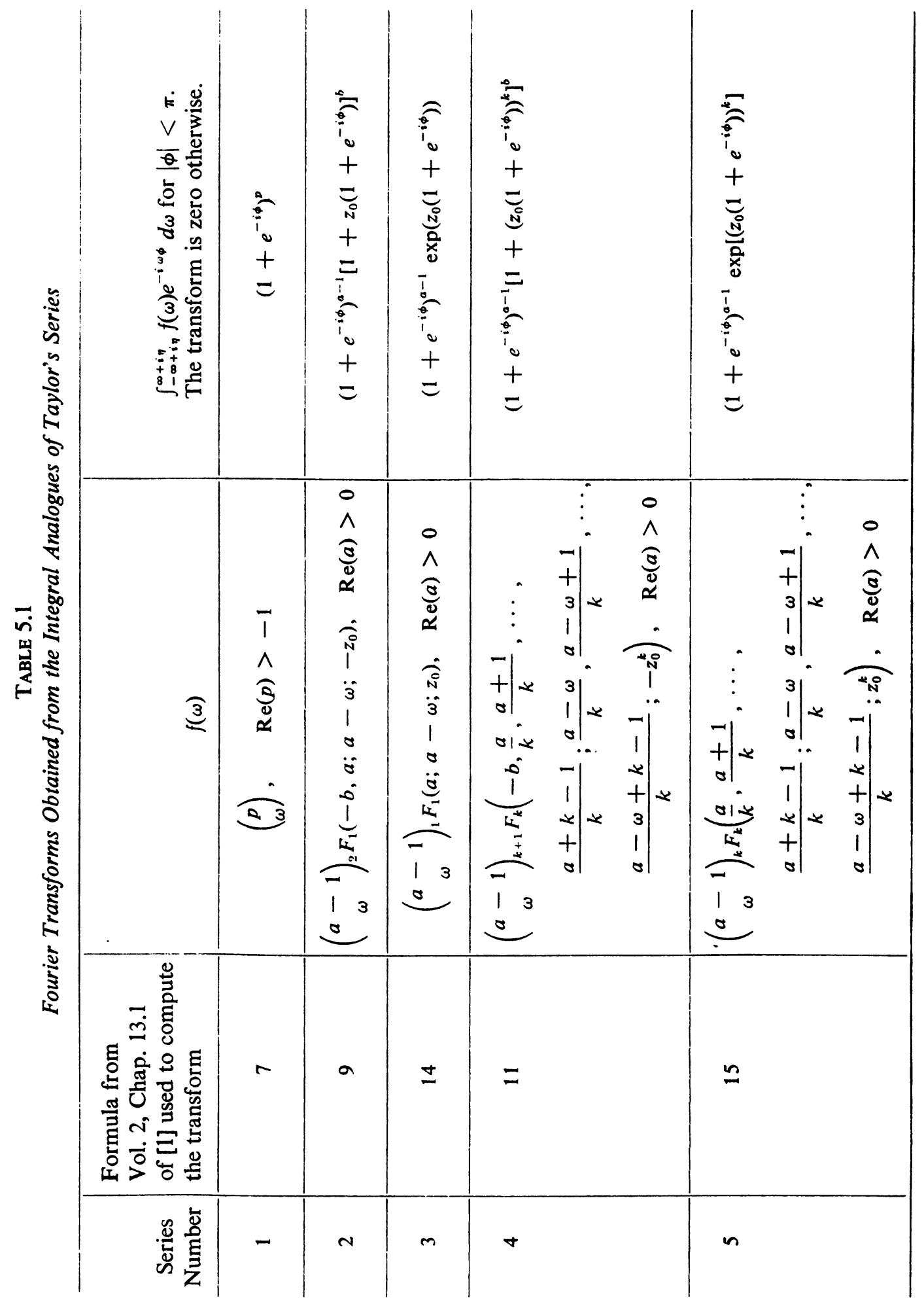


THOMAS J. OSLER

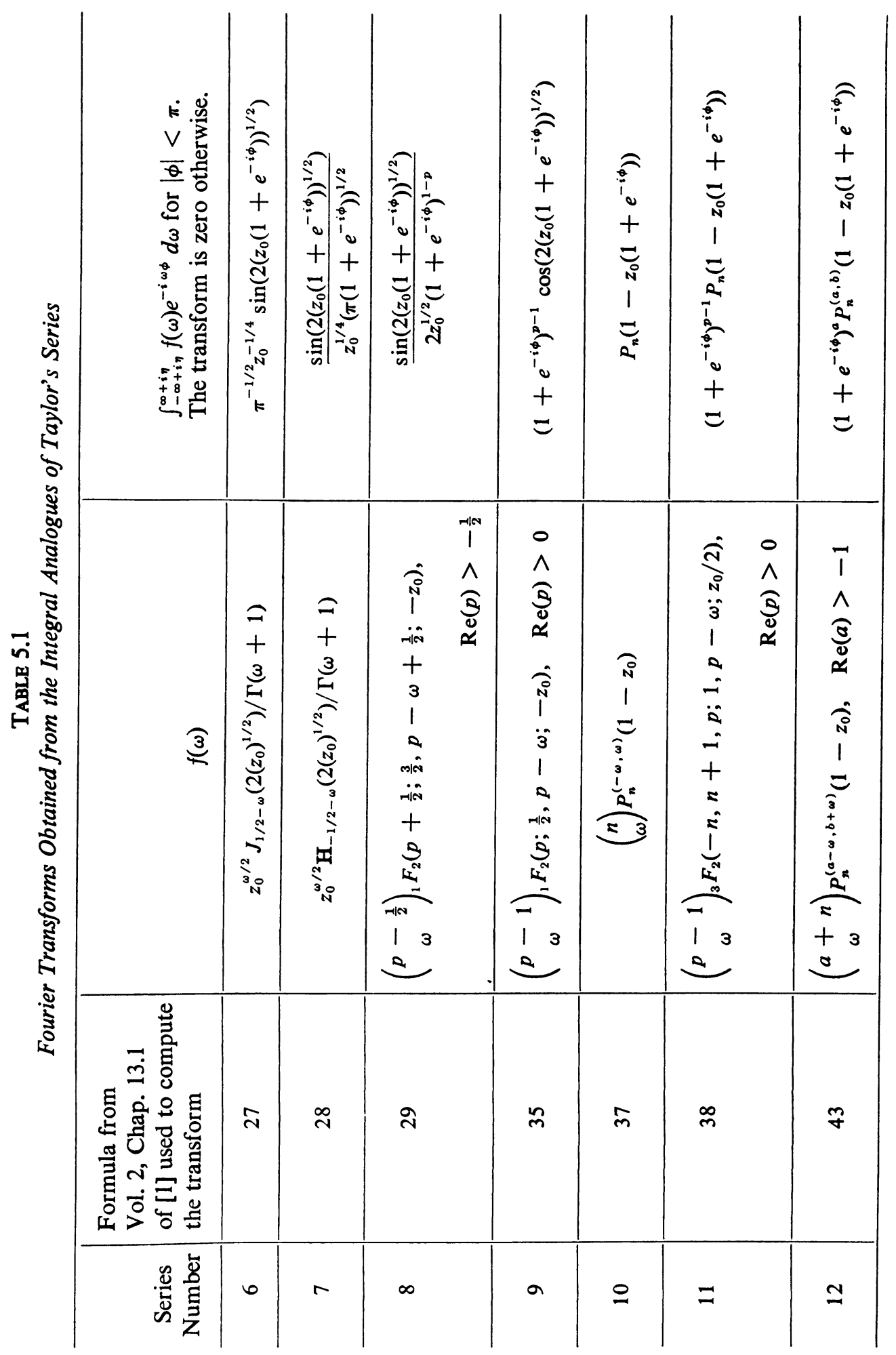


AN INTEGRAL ANALOGUE OF TAYLOR'S SERIES

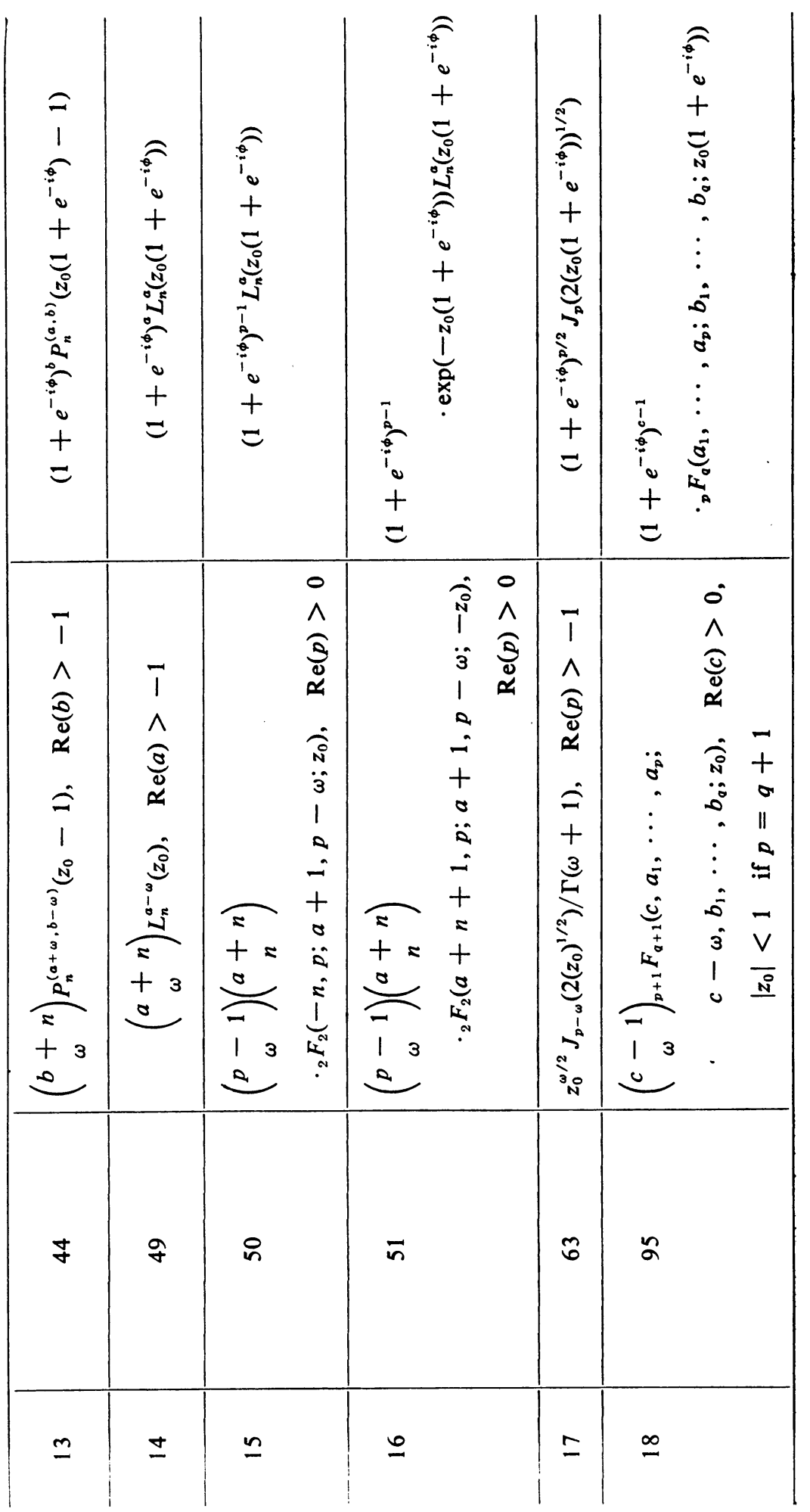




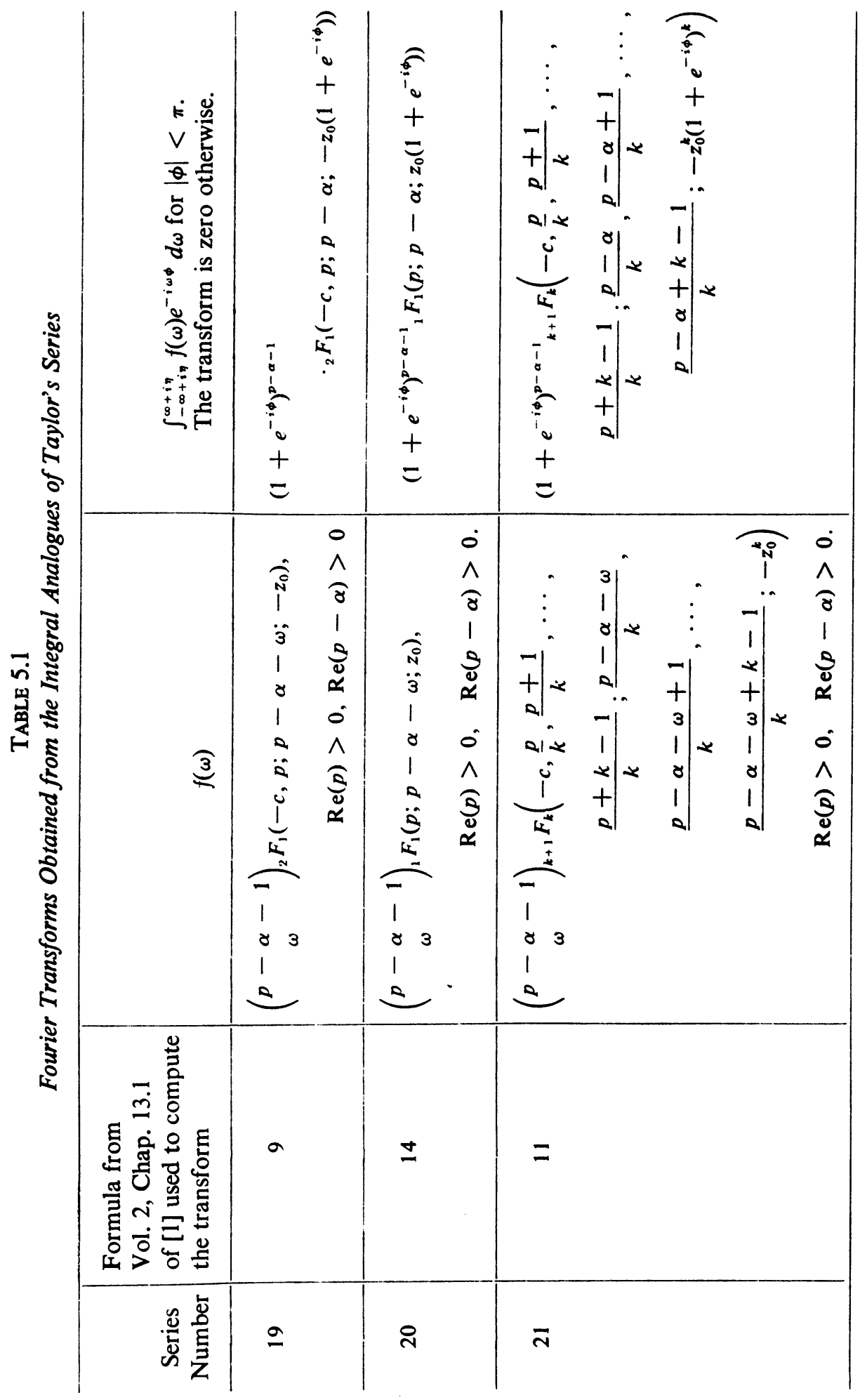




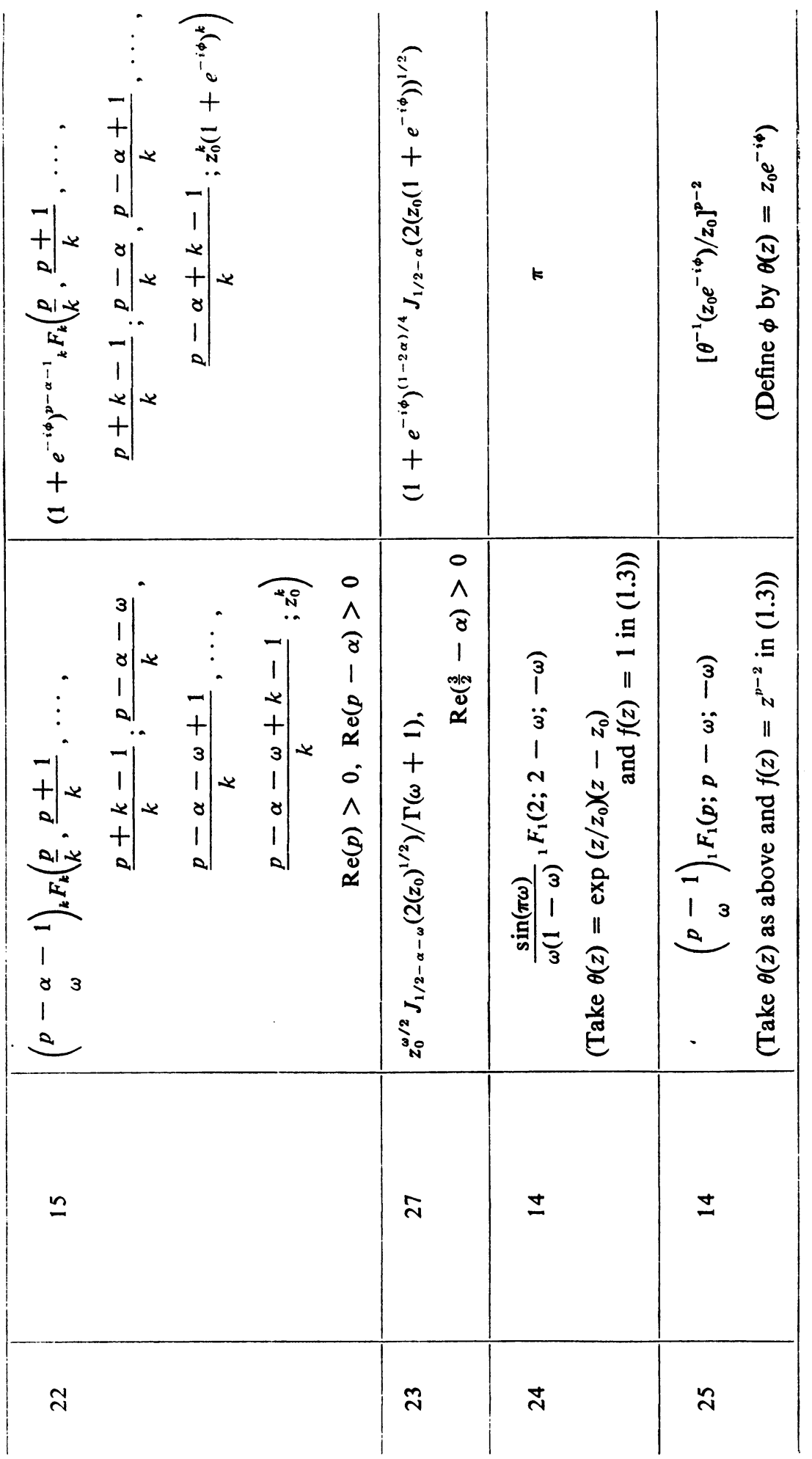




$$
\begin{aligned}
\int_{-\infty+i \eta}^{\infty+i \eta}\left(\begin{array}{l}
p \\
\omega
\end{array}\right) e^{-i \phi \omega} d \omega & =\left(1+e^{-i \phi}\right)^{p}, & & |\phi|<\pi, \\
& =0, & & |\phi|>\pi .
\end{aligned}
$$

Thus we see that our integral analogue of Taylor's series is of value in computing Fourier transforms.

Table 5.1 lists the results of repeating the computation just performed with other functions. The second column lists the formula from Chapter 13.1 of [1, Vol. 2] used to compute the fractional derivatives encountered. Entries 1 through 18 in Table 5.1 are special cases of (1.1), entries 19 through 23 are special cases of (1.2), while entries 24 and 25 are derived from (1.3). The notation for the special functions is that of Erdélyi et al. [1].

Each of the Fourier transformations in Table 5.1 can also be expressed as an infinite sum. We know from [5] that in the r.h.s. of (1.1), (1.2), and (1.3) we can replace

$$
\int_{-\infty}^{\infty} \text { by } \sum_{n=-\infty}^{\infty} \text {, } \omega \text { by } a n \text {, and } d \omega \text { by } a, \text { for } 0<a \leqq 1 \text {. }
$$

This means that the entries in the last column of Table 5.1 can be viewed not only as the Fourier transforms of the corresponding values of $f(\omega)$, but also as

$$
\sum_{n=-\infty}^{\infty} f(a n+\gamma) e^{-i \phi(a n+\gamma)} a,
$$

where $0<a \leqq 1$, and $\gamma$ is an arbitrary complex number.

Department of Mathematics

Rensselaer Polytechnic Institute

Troy, New York 12181

1. A. ERdélyi ET AL., Tables of Integral Transforms. Vols. I, II, McGraw-Hill, New York, 1954. MR 15, 868; MR 16, 468.

2. G. H. HARDY, "Riemann's form of Taylor's series," J. London Math. Soc., v. 20, 1945, pp. 48-57. MR 8, 65 .

3. T. J. OsLER, "Leibniz rule for fractional derivatives generalized and an application to infinite series," SIAM J. Appl. Math., v. 18, 1970, pp. 658-674. MR 41 \#5562.

4. T. J. OsLer, "The fractional derivative of a composite function," SIAM J. Math. Anal., v. 1, 1970, pp. 288-293. MR 41 \#563.

5. T. J. OSLER, "Taylor's series generalized for fractional derivatives and applications," SIAM J. Math. Anal., v. 2, 1971, pp. 37-48.

6. T. J. OsLeR, "Fractional derivatives and Leibniz rule," Amer. Math. Monthly, v. 78, 1971 , pp. $645-649$.

7. T. J. OsLER, "A further extension of Leibniz rule to fractional derivatives and its relation to Parseval's formula," SIAM J. Math. Anal., v. 3, 1972. 